\title{
Determination of Optimal Technological Parameters of a Compaction Process: Case Study
}

\author{
E. Jarošová ${ }^{1}$, E. Kureková ${ }^{2}$ \\ ${ }^{1}$ Department of Logistic and Quality Control, \\ ŠKODA-AUTO University, Tř. V. Klementa 869, 29360 Mladá Boleslav, Czech Republic, jarosova@is.savs.cz \\ ${ }^{2}$ Institute of automation, measurement and applied informatics, \\ Faculty of Mechanical Engineering, Slovak University of Technology, Bratislava, Slovakia, eva.kurekova@stuba.sk
}

\begin{abstract}
Pelletizing as a complicated compaction process is under continuous improvement. One of the problems - determination of optimal technological parameters to attain a sufficiently high density of pellets - is solved in this paper. The statistical model of density depending on four technological factors is built based on data obtained through a central composite design. Canonical analysis is used to find the stationary point, and as the received point is a saddle point, the optimal setting is determined by means of ridge analysis. Special attention is paid to the uncertainty associated with the indirect measurement of the pellet density. Substantial differences in the density exist between pellets created under the same conditions, and especially the type-A uncertainty must be taken into consideration
\end{abstract}

Keywords: Central composite design, response surface methodology, ridge analysis, measurement uncertainties, pressing process

\section{INTRODUCTION}

$\mathrm{H}$ IGH compaction pressing of material is a common feature of all pressing technologies (pelleting, compacting, briquetting). A large number of pressing machines exists, namely for processing biomass into different lengths and shapes. Many factors affect the quality of the resulting product. They can be divided into influences that can be avoided before pressing (e.g., environmental influences, human factor, technological condition of the machine used) and the influences that can be changed or controlled during the pressing process (type of the pressed material, size and humidity of the fraction, internal conditions on the machine).

No technical standards describing or defining precisely products pressed out of organic materials exist in Slovakia. Standards for pressed products (namely pellets) are available only in several European countries like Germany, Austria, or Sweden. Austrian standards ÖNORM M 7135, ÖNORM M 7136 and ÖNORM M 7137, as well as German standards DIN 51731 [1] and DIN PLUS: 2002 [2] related to pellets are generally accepted in Europe. To define the quality of products, those standards describe both mechanical parameters (density, powder density, mechanical resistance) and chemical-thermal parameters (heating capacity, amount of water, ashes and other materials). Beside those parameters, the standards also set shapes and dimensions of pressed products as well as procedures for their testing. But they do not specify limits (tolerances) of technological parameters that should be kept during the production.

Setting of technological parameters affects the resulting properties of pellets. Several papers have been published that introduce simple mathematical models describing the behavior of pressed products made out of different organic materials, e.g., hay [3], or biomass [4]. Optimal setting of technological parameters during the pressing process is continuously researched at the Faculty of Mechanical Engi- neering, Slovak University of Technology in Bratislava as well. Here the pressing machine with adjustable parameters is employed. A number of experiments have been carried out using different varieties of wood. For example, data from runs with pine and oak wood were used in $[5,6,7]$ to study effects of some technological parameters on pellet quality.

The density of the resulting product is a decisive aspect of its quality. The density should reach 1.0 to $1.4 \mathrm{~kg} / \mathrm{dm}^{3}$ (as given by the standard). The statistical model of the dependence of density on some technological parameters enables to predict the density of pellets for different technological conditions.

Our work is a follow-up to the research mentioned above. Data come from [5], where effects of pressure, temperature, moisture content, and particle size fraction on the density were examined by means of an experiment for response surface modeling using the central composite design (CCD).

In our paper, the optimal conditions for achieving maximum density of pine wood pellets are found using canonical and ridge analysis. Considering the large variation of density values calculated on different pieces of pellets produced under the same experimental conditions, measurement uncertainty was included in the density prediction. Measurement uncertainties were dealt with in some previous issues of Measurement Science Review, lately in [8] or [9]. Especially the former work contains some basic principles used in our paper, too.

\section{MEASUREMENT}

Experiments were carried out in laboratory conditions at room temperature, using the pressing machine. The fraction size had been determined and the humidity of the input material had been measured before the pressing process started. Maximum fraction size of the material was achieved by the use of laboratory sieves. The size of sieve openings 
limited the fraction size of the material. The required humidity was ensured in the laboratory drying chamber BINDER. Digital humidity meter GMH 3830 with maximum permissible error of $\pm 0.2 \%$ was used.

The pressing chamber was filled by input material after achieving the desired process temperature (the thermometer with maximum permissible error of $0.5{ }^{\circ} \mathrm{C}$ was used). The required pressure was adjusted and the material was pressed (pressure gauge with maximum permissible error of 0.4 $\mathrm{MPa}$ was used). Seven pieces of pellets were produced at each setting of controlled technological parameters.

The produced pellets were allowed 24 hours for stabilization. Subsequently the length $L$ and diameter $D$ of each produced pellet were measured, using the Mitutoyo CD $-15 \mathrm{D}$ caliper (permissible error of $0.02 \mathrm{~mm}$ ). This allowed the calculation of the volume $V$ of the pellet. The digital balance KERN EW 4200 - 2NM (permissible error $0.02 \mathrm{~g}$ ) was used to determine the mass $m$ of pellet, enabling to calculate the density.

\section{EXPERIMENTAL VARIABLES}

Pressure (factor A), temperature (factor B), moisture content (factor C), and particle size fraction (factor D) represent quantitative factors, effect of which is examined by the experiment. In comparison with the range of parameter values being used so far, the distance of factor levels (except for fraction size) was widened.

The model relating density to these quantitative factors can be described by an equation using either the variables $z_{1}$ to $z_{4}$ with natural levels or the coded variables $x_{1}$ to $x_{4}$. Based on the factorial part of the CCD from [5], the natural variables and the coded variables are related by equations

$$
x_{1}=\frac{z_{1}-127}{32} x_{2}=\frac{z_{2}-100}{15} x_{3}=\frac{z_{3}-10}{2} x_{4}=\frac{z_{4}-2.5}{1.5}
$$

Experimental data are shown in Table 1. Columns $z_{1}$ to $z_{4}$ contain actual settings of factors given in their natural units, while columns $x_{1}$ to $x_{4}$ show the same settings after centering and scaling. Response values in the right column represent averages computed from densities of seven pellets.

The first 16 rows of Table 1 show treatments of the $2^{4}$ factorial part of the $\mathrm{CCD}$, the $17^{\text {th }}$ row represents the approximate center point of the spherical experimental region and remaining rows should correspond to the star points of the CCD. As the natural factor levels at treatments no. 17 to 25 had not been chosen properly in the experiment reported in [5], coded levels of $x_{2}$ to $x_{4}$ in these rows differ from the standard design described in the fundamental books $[10,11,12]$ or in papers $[13,14,15,16,17]$. This fact may have some minor effect to the precision of predictions.

\section{ANALYSIS OF EXPERIMENTAL DATA}

Our analysis consists of several steps. At first the factorial part of the experiment is evaluated and most important effects are found (A). After fitting a second-order response surface model based on CCD (B), the stationary point and its nature are determined by canonical analysis (C). As the stationary point is a saddle point, suitable operation conditions are revealed by means of ridge analysis (D). To predict the density at the optimal conditions, measurement uncertainty is calculated (E) and included in the construction of confidence intervals $(\mathrm{F})$.

Table 1. Experimental data

\begin{tabular}{cccccccccc}
\hline & $\mathrm{A}$ & $\mathrm{B}$ & $\mathrm{C}$ & $\mathrm{D}$ & $\mathrm{A}$ & $\mathrm{B}$ & $\mathrm{C}$ & $\mathrm{D}$ & Response \\
row & $z_{1}$ & $z_{2}$ & $z_{3}$ & $z_{4}$ & $x_{1}$ & $x_{2}$ & $x_{3}$ & $x_{4}$ & $y$ \\
\hline 1 & 95 & 85 & 8 & 1 & -1 & -1 & -1 & -1 & 1.135 \\
2 & 159 & 85 & 8 & 1 & 1 & -1 & -1 & -1 & 1.157 \\
3 & 95 & 115 & 8 & 1 & -1 & 1 & -1 & -1 & 1.191 \\
4 & 159 & 115 & 8 & 1 & 1 & 1 & -1 & -1 & 1.236 \\
5 & 95 & 85 & 12 & 1 & -1 & -1 & 1 & -1 & 0.800 \\
6 & 159 & 85 & 12 & 1 & 1 & -1 & 1 & -1 & 1.007 \\
7 & 95 & 115 & 12 & 1 & -1 & 1 & 1 & -1 & 1.174 \\
8 & 159 & 115 & 12 & 1 & 1 & 1 & 1 & -1 & 1.236 \\
9 & 95 & 85 & 8 & 4 & -1 & -1 & -1 & 1 & 1.089 \\
10 & 159 & 85 & 8 & 4 & 1 & -1 & -1 & 1 & 1.081 \\
11 & 95 & 115 & 8 & 4 & -1 & 1 & -1 & 1 & 1.167 \\
12 & 159 & 115 & 8 & 4 & 1 & 1 & -1 & 1 & 1.206 \\
13 & 95 & 85 & 12 & 4 & -1 & -1 & 1 & 1 & 0.755 \\
14 & 159 & 85 & 12 & 4 & 1 & -1 & 1 & 1 & 0.960 \\
15 & 95 & 115 & 12 & 4 & -1 & 1 & 1 & 1 & 1.128 \\
16 & 159 & 115 & 12 & 4 & 1 & 1 & 1 & 1 & 1.135 \\
\hline 17 & 127 & 100 & 10 & 2 & 0 & 0 & 0 & -0.3 & 1.016 \\
18 & 63 & 100 & 10 & 2 & -2 & 0 & 0 & -0.3 & 1.023 \\
19 & 191 & 100 & 10 & 2 & 2 & 0 & 0 & -0.3 & 1.101 \\
20 & 127 & 55 & 10 & 2 & 0 & -3 & 0 & -0.3 & 0.972 \\
21 & 127 & 130 & 10 & 2 & 0 & 2 & 0 & -0.3 & 1.146 \\
22 & 127 & 100 & 5 & 2 & 0 & 0 & -2.5 & -0.3 & 1.186 \\
23 & 127 & 100 & 15 & 2 & 0 & 0 & 2.5 & -0.3 & 1.053 \\
24 & 127 & 100 & 10 & 0.5 & 0 & 0 & 0 & -1.3 & 1.024 \\
25 & 127 & 100 & 10 & 4 & 0 & 0 & 0 & 1 & 1.012 \\
\hline & & & & & & & & &
\end{tabular}

\section{A. Analysis of the factorial part of the experiment}

The factorial part $2^{4}$ of the CCD experiment enables to build a model with interactions. Only two-factor interactions were considered in response surface modeling. The model using coded variables has the form

$$
y=\beta_{0}+\beta_{1} x_{1}+\beta_{2} x_{2}+\ldots+\beta_{12} x_{1} x_{2}+\ldots+\beta_{34} x_{3} x_{4}+\varepsilon
$$

where $\beta_{0}, \beta_{1}$ etc. are unknown parameters. Besides, $\beta_{1}$ represents, at the same time, one half of the main effect of factor $A, \beta_{12}$ equals to one half of the interaction $\mathrm{AB}$ effect, etc. in the coding used.

The analysis of variance was performed with the statistical 
packet SAS. Only effects A, B, C, and the interaction BC were significant with $\mathrm{p}$-value less than 0.05 (details are not shown). The importance of different factors can be evaluated through partition of the total sum of squares by the factors (Table 2). At this stage of knowledge, excluding factor D from the next experimentation would seem reasonable, but in spite of it, all four factors were comprised in the augmented experiment in [5].

Table 2. Partition sum of squares by factors, $2^{4}$ factorial, PROC RSREG

\begin{tabular}{|c|c|c|c|c|c|}
\hline Factor & DF & Sum of Squares & Mean Square & F Value & Pr $>$ F \\
\hline A & 4 & 0.035319 & 0.008830 & 3.66 & 0.0936 \\
\hline B & 4 & 0.184549 & 0.046137 & 19.13 & 0.0031 \\
\hline C & 4 & 0.121882 & 0.030471 & 12.64 & 0.0080 \\
\hline D & 4 & 0.011563 & 0.002891 & 1.20 & 0.4140 \\
\hline
\end{tabular}

\section{B. Analysis of the Central Composite Experiment}

Considering four factors, the central composite design arises by combining the original $2^{4}$ factorial design with 8 star points at the distance $\pm \alpha$ from the center $(0,0,0,0)$ (in coded units) of the experimental region. Moreover, two or more runs at $(0,0,0,0)$ are commonly replicated. To achieve the same accuracy of predictions at the same distance from the center, $\alpha=\sqrt[4]{2^{4}}=2$ is chosen (Table 3 ), see $[10,11$, $12]$.

Table 3. Star points in coded units

\begin{tabular}{cccc}
\hline $\mathrm{A}$ & $\mathrm{B}$ & $\mathrm{C}$ & $\mathrm{D}$ \\
$x_{1}$ & $x_{2}$ & $x_{3}$ & $x_{4}$ \\
\hline-2 & 0 & 0 & 0 \\
2 & 0 & 0 & 0 \\
0 & -2 & 0 & 0 \\
0 & 2 & 0 & 0 \\
0 & 0 & -2 & 0 \\
0 & 0 & 2 & 0 \\
0 & 0 & 0 & -2 \\
0 & 0 & 0 & 2 \\
\hline
\end{tabular}

Comparing Table 3 with the corresponding rows of Table 1 implies that some star points and the center point were not chosen properly. In spite of this, the second-order model corresponding to model (2) with added second-order terms can be constructed

$$
y=\beta_{0}+\beta_{1} x_{1}+\ldots+\beta_{12} x_{1} x_{2}+\ldots+\beta_{11} x_{1}^{2}+\ldots+\beta_{44} x_{4}^{2}+\varepsilon
$$

Again, results in Table 4 clearly confirm uselessness of factor D. After excluding D and relating interactions, a new model was fitted. Details are given in Table 5. As can be seen, particularly the effects of B and C and their interaction are important. The mean square error of the fitted response surface read from the output of PROC RSREG (not displayed) which will be needed for interval estimation, was $S S_{E} /(n-p)=0.057099 /(25-10)=0.003807$, where $S S_{E}$ denotes the residual sum of squares, $n$ the number of observations and $p$ the number of model parameters (rows in Table 5).

Table 4. Partition sum of squares by factors, CCD, PROC RSREG

\begin{tabular}{|c|c|c|c|c|c|}
\hline Factor & DF & Sum of Squares & Mean Square & F Value & Pr $>$ F \\
\hline A & 5 & 0.045557 & 0.009111 & 2.30 & 0.1228 \\
\hline B & 5 & 0.194773 & 0.038955 & 9.83 & 0.0013 \\
\hline C & 5 & 0.141049 & 0.028210 & 7.12 & 0.0044 \\
\hline D & 5 & 0.017490 & 0.003498 & 0.88 & 0.5264 \\
\hline
\end{tabular}

Table 5. Estimated parameters, PROC RSREG

\begin{tabular}{|c|c|c|c|r|c|}
\hline Parameter & DF & Estimate & Standard Error & $\boldsymbol{t}$ Value & Pr $>|\boldsymbol{t}|$ \\
\hline Intercept & 1 & 1.031108 & 0.028078 & 36.72 & $<.0001$ \\
\hline $\mathrm{A}$ & 1 & 0.030625 & 0.012594 & 2.43 & 0.0280 \\
\hline $\mathrm{B}$ & 1 & 0.076036 & 0.012453 & 6.11 & $<.0001$ \\
\hline $\mathrm{C}$ & 1 & -0.049105 & 0.011557 & -4.25 & 0.0007 \\
\hline $\mathrm{A} * \mathrm{~B}$ & 1 & -0.017062 & 0.015424 & -1.11 & 0.2861 \\
\hline $\mathrm{A} * \mathrm{C}$ & 1 & 0.023938 & 0.015424 & 1.55 & 0.1415 \\
\hline $\mathrm{B} * \mathrm{C}$ & 1 & 0.050812 & 0.015424 & 3.29 & 0.0049 \\
\hline $\mathrm{A} * \mathrm{~A}$ & 1 & 0.014125 & 0.013923 & 1.01 & 0.3264 \\
\hline $\mathrm{B} * \mathrm{~B}$ & 1 & 0.016259 & 0.008312 & 1.96 & 0.0693 \\
\hline $\mathrm{C} * \mathrm{C}$ & 1 & 0.016765 & 0.008807 & 1.90 & 0.0763 \\
\hline
\end{tabular}

\section{Canonical Analysis}

After fitting the response surface, the location and nature of the stationary point were determined. In matrix notation, the fitted model has the form

$$
\hat{y}=b_{0}+\boldsymbol{x}^{T} \boldsymbol{b}+\boldsymbol{x}^{T} \hat{\boldsymbol{B}} \boldsymbol{x}
$$

where

$\boldsymbol{x}^{T}=\left(\begin{array}{llllllllll}1 & x_{1} & x_{2} & x_{3} & x_{1} x_{2} & \ldots & x_{2} x_{3} & x_{1}^{2} & \ldots & x_{3}^{2}\end{array}\right)$,

$b_{0}=1.031108$,

$\boldsymbol{b}=\left[\begin{array}{r}0.030625 \\ 0.076036 \\ -0.049105\end{array}\right]$

$\hat{\boldsymbol{B}}=\left[\begin{array}{rrr}0.014125 & -0.008531 & 0.011969 \\ -0.008531 & 0.016259 & 0.025406 \\ 0.011969 & 0.025406 & 0.016765\end{array}\right]$, see Table 5.

The stationary point is

$\boldsymbol{x}_{s}=-\frac{1}{2} \hat{\boldsymbol{B}}^{-1} \boldsymbol{b}=\left[\begin{array}{c}0.763585 \\ 0.820396 \\ -0.790467\end{array}\right]$ (in coded units) 

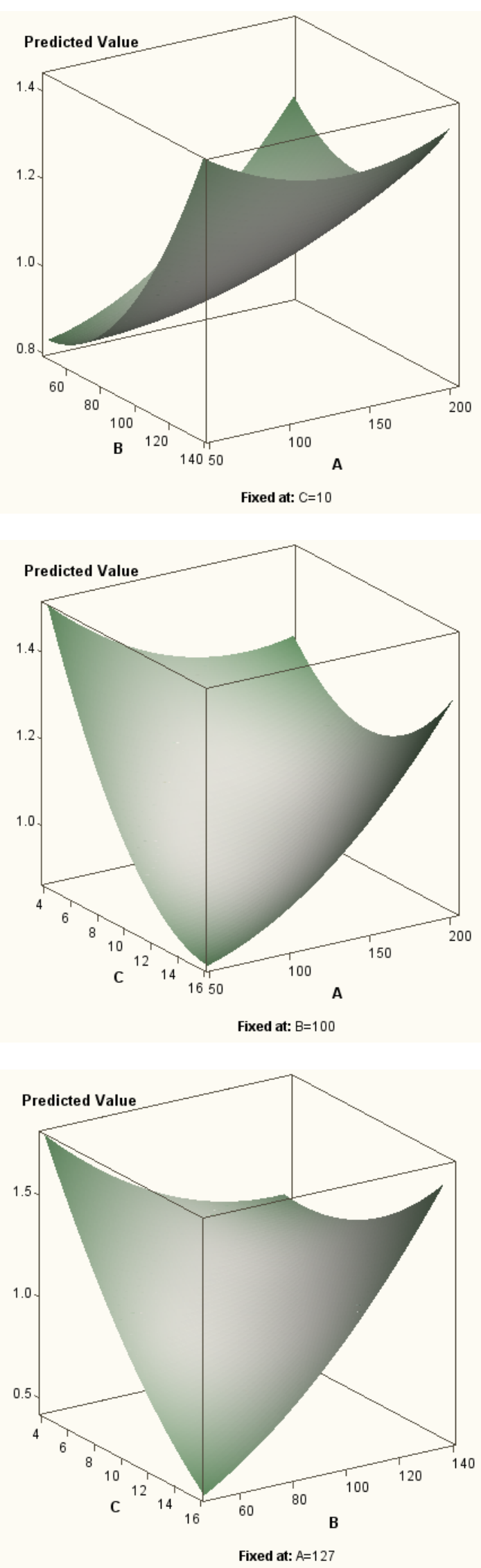

Fig. 1. Fitted quadratic response surfaces or $\mathbf{Z}_{s}=\left[\begin{array}{c}175.9 \\ 123.3 \\ 6.0\end{array}\right]$ (in the natural units, as follows from (1)).

The eigenvalues of the matrix $\hat{\boldsymbol{B}}$ are $\lambda_{1}=0.262823$, $\lambda_{2}=0.091287, \lambda_{3}=-0.091207$. Mixed signs of the eigenvalues imply that the stationary point is a saddle point, see $[11,12]$. The predicted response at the saddle point, i.e. at $z_{1}=176 \mathrm{MPa}, z_{2}=123{ }^{\circ} \mathrm{C}$, and $z_{3}=6 \%$ is 1.162 $\mathrm{kg} / \mathrm{dm}^{3}$.

Three-dimensional surfaces plotted against different pairs of factors are displayed in Fig.1. The third factor is always set to its level at the center point.

\section{Ridge Analysis}

To get a better idea about behaviour of the response in the neighbourhood of the saddle point, ridge analysis was performed using PROC RSREG. It consists in maximization of the predicted response given by (4) subject to the constraint $\boldsymbol{x}_{p}^{T} \boldsymbol{x}_{p}=R^{2}$, where $\boldsymbol{x}_{p}^{T}=\left(\begin{array}{llll}x_{1} & x_{2} & x_{3} & x_{4}\end{array}\right)$ and $R$ is the radius in coded units (details see [11]).

The computed ridge of maximum response for $R \in<0,1>$ is displayed in Fig. 2. It starts at the center point (the value 92.5 of $B$ instead of 100 follows from the improper plan) and goes through points at specified radii corresponding to factor settings that maximize the predicted response at this radius. The predicted response values are displayed on the top, corresponding factor settings can be read on the plot below or in Table 6 . This table indicates that the maximum predicted response at a distance of one coded unit from the center is $\hat{y}=1.329$ with the estimated standard error $s_{\hat{y}}=0.072$ and that this constrained maximum can be achieved approximately at pressure $127 \mathrm{MPa}$, temperature $77^{\circ} \mathrm{C}$, and moisture $5 \%$.

Table 6. Ridge analysis, PROC RSREG, uncoded factor values

\begin{tabular}{|c|c|c|c|c|c|}
\hline \multicolumn{6}{|c|}{ Estimated Ridge of Maximum Response for Variable $\boldsymbol{y}$} \\
\hline \multirow{2}{*}{$\begin{array}{c}\text { Coded } \\
\text { Radius }\end{array}$} & Estimated & Standard & \multicolumn{2}{c|}{ Uncoded Factor Values } \\
\cline { 5 - 6 } & Response & Error & A & B & C \\
\hline 0.0 & 0.99716 & 0.02859 & 127.00000 & 92.50000 & 10.00000 \\
\hline 0.1 & 1.02146 & 0.02848 & 128.83724 & 94.64026 & 9.61534 \\
\hline 0.2 & 1.04426 & 0.02795 & 130.20616 & 96.35116 & 9.17928 \\
\hline 0.3 & 1.06625 & 0.02720 & 130.71395 & 96.51392 & 8.62909 \\
\hline 0.4 & 1.08970 & 0.02711 & 130.37388 & 94.25291 & 8.03127 \\
\hline 0.5 & 1.11706 & 0.02881 & 129.86267 & 91.32654 & 7.51494 \\
\hline 0.6 & 1.14919 & 0.03289 & 129.36111 & 88.41579 & 7.05561 \\
\hline 0.7 & 1.18636 & 0.03946 & 128.87812 & 85.57279 & 6.62726 \\
\hline 0.8 & 1.22866 & 0.04830 & 128.40909 & 82.78418 & 6.21718 \\
\hline 0.9 & 1.27614 & 0.05914 & 127.95002 & 80.03534 & 5.81880 \\
\hline 1.0 & 1.32883 & 0.07174 & 127.49814 & 77.31567 & 5.42839 \\
\hline
\end{tabular}




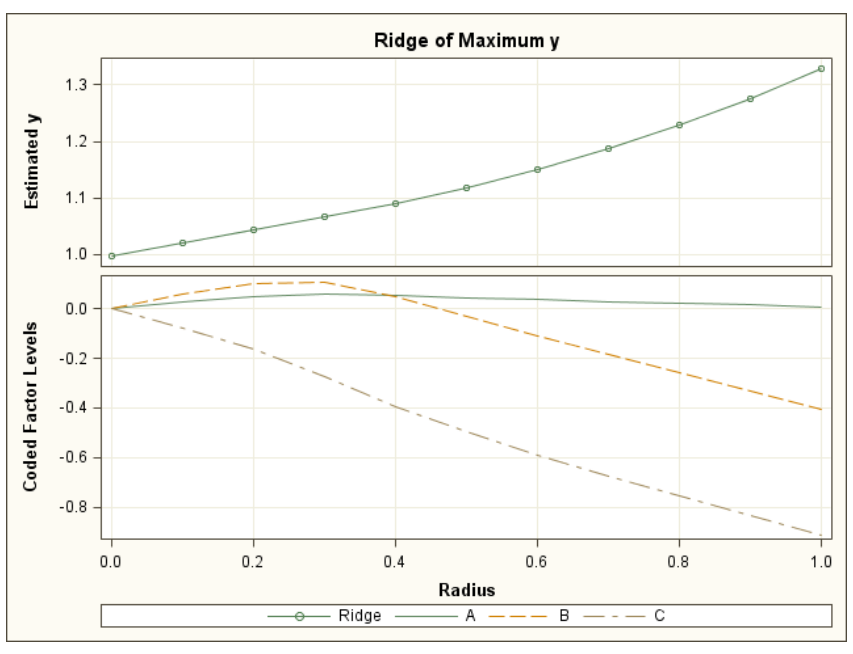

Fig. 2. Maximum response and constrained maximum conditions plotted against radius, PROC RSREG

\section{E. Measurement uncertainty at the center point}

The uncertainty of the estimate of pellet density was calculated for the center point (pressure $127 \mathrm{MPa}$, temperature $100{ }^{\circ} \mathrm{C}$, humidity $10 \%$, fraction size $2 \mathrm{~mm}$ ). Seven pellets were produced (see Table 7).

Table 7. Measured and calculated values at the center point, $s$ denotes the sample standard deviation

\begin{tabular}{|c|c|c|c|c|c|}
\hline $\begin{array}{l}\text { pecimen } \\
\text { No. } \\
\end{array}$ & $\begin{array}{c}\text { Diameter } \\
(\mathbf{d m})\end{array}$ & $\begin{array}{c}\text { Length } \\
\text { (dm) }\end{array}$ & $\begin{array}{c}\text { Mass } \\
(\mathrm{kg})\end{array}$ & $\begin{array}{c}\text { Volume } \\
\left(\mathbf{d m}^{3)}\right.\end{array}$ & \begin{tabular}{|} 
Density \\
$\left(\mathbf{k g} / \mathbf{d m}^{3}\right)$
\end{tabular} \\
\hline 7 & & & & & \\
\hline 2 & & & & & \\
\hline 3 & & & & & \\
\hline 4 & & & & & \\
\hline 5 & & & & & \\
\hline 6 & & & & & \\
\hline 7 & & & & & \\
\hline era & & 0.3 & & 014 & \\
\hline & 0.00022 & 0.07783 & 0.003 & 0.00244 & 0.13681 \\
\hline
\end{tabular}

The following equation was used for calculating the pressed pellet density $\rho=V / m$.

An estimate of the density is represented by the arithmetic mean obtained out of $r=7$ calculated densities of the individual pressed pellets, i.e. $\bar{\rho}=1.015887 \mathrm{~kg} / \mathrm{dm}^{3}$.

The standard uncertainty of the estimate $\bar{\rho}$, calculated by the type A method, can be expressed as

$u_{\mathrm{A}}(\bar{\rho})=\frac{s_{\rho}}{\sqrt{n}}=\frac{0.136808}{\sqrt{7}}=0.051709 \mathrm{~kg} / \mathrm{dm}^{3}$.

When calculating the standard uncertainty determined by the type B method, only the effect of permissible errors of the individual measuring instruments was assumed. As the same measuring instrument was used for measurement of both the length and diameter of the pellet, covariances were included as well. For model $\rho=V / m$ we get

$$
u_{\mathrm{B}}^{2}(\bar{\rho})=C^{2}(m) \cdot u_{\mathrm{B}}^{2}(m)+C^{2}(V) \cdot u_{\mathrm{B}}^{2}(V)
$$

where $C(m)$ and $C(V)$ are sensitivity coefficients

$$
\begin{aligned}
& C(m)=\left(\frac{\partial \rho}{\partial m}\right)=\frac{1}{\bar{V}}=\frac{1}{0.01014}=98.5823258 \mathrm{dm}^{-3} \\
& C(V)=\left(\frac{\partial \rho}{\partial V}\right)=-\frac{\bar{m}}{\bar{V}^{2}}=-\frac{0.01053}{0.01014^{2}}=-102.336 \mathrm{~kg} / \mathrm{dm}^{2}
\end{aligned}
$$

The uniform probability distribution is assumed for the digital balance KERN EW 4200 - 2NM, while the maximum permissible error $\Delta_{\text {per }}$, stated in certificate, reaches $0.02 \mathrm{~g}$. Then $u_{\mathrm{B}}(m)=\Delta_{\mathrm{per}} / \sqrt{3}=1.1547 \cdot 10^{-5} \mathrm{~kg}$.

When determining $u_{\mathrm{B}}(V)$, we employ the model of volume $V$ measurement in the form $V=\pi D^{2} L / 4$.

Then [18]

$$
\begin{aligned}
u_{\mathrm{B}}^{2}(V)= & C^{2}(D) \cdot u_{\mathrm{B}}^{2}(D)+C^{2}(L) \cdot u_{\mathrm{B}}^{2}(L)+ \\
& +2 C(D) \cdot C(L) \cdot u_{\mathrm{B}}(D, L)
\end{aligned}
$$

where sensitivity coefficients $C(D)$ and $C(L)$ are

$C(D)=\left(\frac{\partial V}{\partial D}\right)=\frac{2 \pi \bar{D}}{4} \bar{L}=\frac{\pi \cdot 0.2004 \cdot 0.32186}{2}=$

$=0.101265 \mathrm{dm}^{2}$

$C(L)=\left(\frac{\partial V}{\partial L}\right)=\frac{\pi \cdot \bar{D}^{2}}{4}=\frac{\pi \cdot 0.2004^{2}}{4}=0.031526 \mathrm{dm}^{2}$

The length $L$ and the diameter $D$ of the pressed products were measured by the same instrument Mitutoyo CD - 15D, then $u_{\mathrm{B}}(D)=u_{\mathrm{B}}(L)$ and they can be evaluated according to the measuring instrument certificate which reads maximum permissible error of $0.02 \mathrm{~mm}$. Based on a large number of experiments carried out on the digital caliper, it has shown that errors of calipers behave according to normal distribution regardless of the manufacturer and the calibration point [19]. Then for the containment probability $95 \%, u_{\mathrm{B}}(D)=u_{\mathrm{B}}(L)=\Delta_{\mathrm{per}} / 2=1 \cdot 10^{-4} \mathrm{dm}$.

As both the pressed product diameter and length were measured by the same instrument, the data are correlated and the correlation is calculated as

$u_{\mathrm{B}}(D, L)=r_{L, D} \cdot u_{\mathrm{B}}(D) u_{\mathrm{B}}(L)$

While the error of measuring instrument is considered as the only uncertainty source, we assume $r_{L, D}=1$. Then $u_{\mathrm{B}}(D, L)=1 \cdot u_{\mathrm{B}}(D) u_{\mathrm{B}}(L)=1 \cdot 10^{-8} \mathrm{dm}^{2}$.

After substituting to (6) we get 
$u_{\mathrm{B}}^{2}(V)=C^{2}(D) \cdot u_{\mathrm{B}}^{2}(D)+C^{2}(L) \cdot u_{\mathrm{B}}^{2}(L)+$

$+2 C(D) \cdot C(L) \cdot u_{\mathrm{B}}(D, L)=1.76334 \cdot 10^{-10} \mathrm{~kg}^{2} / \mathrm{dm}^{6}$

and subsequently to the equation (5)

$u_{\mathrm{B}}^{2}(\bar{\rho})=C^{2}(m) \cdot u_{\mathrm{B}}^{2}(m)+C^{2}(V) \cdot u_{\mathrm{B}}^{2}(V)=3.1425 \cdot 10^{-6}$

$\mathrm{kg}^{2} / \mathrm{dm}^{6}$.

The combined standard uncertainty $u_{\mathrm{C}}$ of the pressed product density is calculated as

$u_{\mathrm{C}}(\bar{\rho})=\sqrt{u_{\mathrm{A}}^{2}(\bar{\rho})+u_{\mathrm{B}}^{2}(\bar{\rho})}=0.051709 \approx 0.052 \mathrm{~kg} / \mathrm{dm}^{3}$.

It seems obvious that uncertainty of measurement at the center point is caused only by variation of measured data, thus $u_{\mathrm{C}}(\bar{\rho}) \approx u_{\mathrm{A}}(\bar{\rho})$. Type-A uncertainty at the other experimental points can be determined accordingly, with the use of sample variances given in Table 8. As the Levene's test for assessing variance homogeneity is not significant ( $p$ value as high as 0.0570$)$, the sample variances at all 25 experimental points can be pooled by taking the average variance of 0.006638 . Then the overall measurement uncertainty $u_{\mathrm{A}}(\bar{\rho})$ can be set to $\sqrt{0.006638 / 7}=0.030795$. This uncertainty is considered in construction of prediction limits.

\section{F. Prediction interval}

$100(1-\alpha) \%$ prediction interval for $\bar{\rho}$ at the optimal operational conditions is constructed according to the formula known from regression analysis

$$
\hat{y}_{o} \pm t_{1-\alpha / 2}\left(v_{e f}\right) \hat{\sigma} \sqrt{1+\mathbf{x}_{o}^{T}\left(\mathbf{X}^{T} \mathbf{X}\right)^{-1} \mathbf{x}_{o}}
$$

where $\hat{y}_{o}$ denotes the estimated optimal density $1.328825, \boldsymbol{X}$ is the design matrix of type $25 \times 10, \mathbf{x}_{o}^{T}$ is the row vector ( $1127.49877 .3165 .428 \ldots ..), \hat{\sigma}$ is the estimated standard deviation of the random component $\varepsilon$ in model (3) and $t_{1-\alpha / 2}(v)$ denotes the upper percentile of the Student distribution with $v$ degrees of freedom that correspond to the way of $\sigma$ estimation. Unlike in common CCD applications, the measurement uncertainty cannot be neglected and is added to the experimental error estimated by means of $\hat{\sigma}_{E}^{2}=S S_{E} /(n-p)$ (see paragraph B), i.e.

$$
\hat{\sigma}^{2}=\hat{\sigma}_{E}^{2}+u_{\mathrm{A}}^{2}
$$

The effective degrees of freedom are given by WelchSatterthwaite equation [20], i.e.

$$
v_{e f} \approx \hat{\sigma}^{4} /\left(\hat{\sigma}_{E}^{4} / v_{1}+u_{\mathrm{A}}^{4} / v_{2}\right),
$$

where $v_{1}=n-p=25-10=15, \quad v_{2}=25(r-1)=150$. Accordingly, $\hat{\sigma}^{2}=0.003807+0.000948=0.004755, v_{e f} \approx 23$, $t_{0.975}(23)=2.069$, and the $95 \%$ prediction interval is $(1.110 ; 1.548)$. As can be seen, the lower prediction limit is higher than $1 \mathrm{~kg} / \mathrm{dm}^{3}$ and so the requirement on the sufficiently high density is met.

Table 8. Characteristics of density at experimental points

\begin{tabular}{ccccccc}
\hline & $\mathrm{A}$ & $\mathrm{B}$ & $\mathrm{C}$ & $\mathrm{D}$ & & \\
row & $z_{1}$ & $z_{2}$ & $z_{3}$ & $z_{4}$ & $\bar{y}$ & $s^{2}$ \\
\hline 1 & 95 & 85 & 8 & 1 & 1.135 & 0.001258 \\
2 & 159 & 85 & 8 & 1 & 1.157 & 0.001384 \\
3 & 95 & 115 & 8 & 1 & 1.191 & 0.002513 \\
4 & 159 & 115 & 8 & 1 & 1.236 & 0.001332 \\
5 & 95 & 85 & 12 & 1 & 0.800 & 0.00727 \\
6 & 159 & 85 & 12 & 1 & 1.007 & 0.009572 \\
7 & 95 & 115 & 12 & 1 & 1.174 & 0.007584 \\
8 & 159 & 115 & 12 & 1 & 1.236 & 0.006393 \\
9 & 95 & 85 & 8 & 4 & 1.089 & 0.001384 \\
10 & 159 & 85 & 8 & 4 & 1.081 & 0.002974 \\
11 & 95 & 115 & 8 & 4 & 1.167 & 0.003207 \\
12 & 159 & 115 & 8 & 4 & 1.206 & 0.001062 \\
13 & 95 & 85 & 12 & 4 & 0.755 & 0.006349 \\
14 & 159 & 85 & 12 & 4 & 0.960 & 0.006146 \\
15 & 95 & 115 & 12 & 4 & 1.128 & 0.004268 \\
16 & 159 & 115 & 12 & 4 & 1.135 & 0.001377 \\
\hline 17 & 127 & 100 & 10 & 2 & 1.016 & 0.018716 \\
18 & 63 & 100 & 10 & 2 & 1.023 & 0.008629 \\
19 & 191 & 100 & 10 & 2 & 1.101 & 0.020944 \\
20 & 127 & 55 & 10 & 2 & 0.972 & 0.006323 \\
21 & 127 & 130 & 10 & 2 & 1.146 & 0.003376 \\
22 & 127 & 100 & 5 & 2 & 1.186 & 0.002007 \\
23 & 127 & 100 & 15 & 2 & 1.053 & 0.017137 \\
24 & 127 & 100 & 10 & 0.5 & 1.024 & 0.014276 \\
25 & 127 & 100 & 10 & 4 & 1.012 & 0.010476 \\
\hline & & & & & &
\end{tabular}

\section{DISCUSSION}

As was already mentioned, the design of the experiment in [5] slightly differred from the CCD recommended in literature. Natural factor levels in the augmented part of the CCD experiment were not in agreement with $\alpha=2$ and replicated measurements at the center point were not conducted. This fact may affect more the accuracy of estimates than the result in terms of optimal conditions. It should be also noted that the spherical CCD used in [5] is not the only possible design; the cuboidal CCD can be used where the factor levels from the factorial part cannot be 
exceeded (see [11]). A special remark should be made about the particle size fraction; the particle size fraction cannot be controlled entirely in the experiment because only its upper limit is guaranteed and so different factor levels are overlapping.

As for the evaluation of experimental data, it needs to be emphasised that seven measurements at each experimental point are not true replications because they are obtained by a single experimental setting. This situation is usually handled as was shown in this paper, i.e. averages from samples of size seven are evaluated instead of individual response values. Then a negligible measurement uncertainty is assumed, though. It is not the case here and so we contemplated two approaches. The first consists in treating individual measurements as replications and neglecting measurement uncertainties, the other in treating averages and taking measurement uncertainties into consideration. The former approach is not recommended for the reason that a great number of pseudoreplications imply that many tested effects may falsely appear significant.

In the latter approach, determination of the measurement uncertainty is required. In the analysis of CCD experiments the experimental error is estimated by means of runs replicated at the center point. We mimicked this approach and calculated the combined measurement uncertainty at this point. As the type-B uncertainty was negligible in comparison with the type-A, only A-uncertainties were taken into account and averaged over all twenty five experimental points.

To decrease the type-A uncertainty of density (and so a more precise prediction based on the model), causes of the large variation should be found. One of them might be the not truly cylindrical shape of pellets and in such case a more sophisticated measurement of their dimensions should be designed.

Determination of the stationary point is realizable by means of most statistical software products. Application of ridge analysis assumes either suitable statistical software (SAS, R etc.) or a macro for constrained optimization. Modification of the formula for the prediction interval follows from basic statistical principles.

\section{CONCLUSIONS}

At present, the trial and error method is often used when technological parameters in compaction processes are set. The paper shows how data from the suitably designed experiment can be used to find the operational conditions that ensure the optimal density of pellets, and represents a significant contribution to the research in this field.

With the present method of density indirect measurement, the type-A measurement uncertainty, beside the experimental error, must be considered for prediction based on the response surface model. To verify whether the predicted density is high enough, the lower prediction limit is used. It guarantees (at the considered confidence level) that the real density at the given setting measured in the same way, i.e. expressed as the average from the sample of the same size as was used in the experiment, will not be lower than the limit.

\section{ACKNOWLEDGMENT}

This work was supported by the Agency of the Ministry of Education of the Slovak Republic, VEGA grant No. 1/0120/12 and by SKODA AUTO a.s., IGA/2012/9.

\section{REFERENCES}

[1] DIN 51731:1996 Testing of solid fuels - compressed untreated wood, requirements and testing. Berlin, Germany: Deutsches Institut für Normung

[2] DIN PLUS: 2002 Certification Scheme. Wood pellets for use in small furnaces. Berlin, Germany. DIN CERTCO - Gesellschaft für Konformitätsbewertung $\mathrm{mbH}$

[3] Mani, S., Tabil, L. G., Sokhansanj, S. (2006). Effects of compressive force, particle size and moisture content on mechanical properties of biomass pellets from grasses. Biomass and Bioenergy 30 (7), 648-654

[4] Križan, P., Šooš, L', Matúš, M. (2010). Optimalisation of briquetting machine pressing chamber geometry. Machine Design. s. 19-24

[5] Svátek, M. (2010). Vplyv vybraných parametrov na kvalitu výliskov. (In Slovak). Unpublished doctoral dissertation, Slovak University of Technology, Bratislava, Slovak Republic.

[6] Svátek, M., Križan, P., Šooš, L., Kureková, E. (2009). Education of parameter impact on final briquettes quality. In Proceedings of the 7th International Conference on Measurement. Smolenice, Slovak Republic.

[7] Križan, P., Šooš, L', Matúš, M., Svátek, M., Vukelič, D. (2010). Evaluation of measured data from research of parameters impact on final briquettes density. Aplimat - Journal of Applied Mathematics 3 (3), 6876

[8] Kosarevsky, S.V., Latypov, V.N. (2012): Practical Procedure for Position Tolerance Uncertainty Determination via Monte-Carlo Error Propagation. Measurement Science Review 12 (1), 1-7.

[9] Zhang, F., Qu, X. (2012). Fusion Estimation of Point Sets from Multiple Stations of Spherical Coordinate Instruments Utilizing Uncertainty Estimation Based on Monte Carlo. Measurement Science Review 12 (2), 40-45.

[10] Likeš, J. (1968). Navrhování průmyslových experimentů. (in Czech) Praha: SNTL.

[11] Myers, R.H., Montgomery, D.C. (2002). Response Surface Methodology: Process and Product Optimization Using Designed Experiments. New York: John Wiley \& Sons.

[12] Wu, C.F.J., Hamada, M. (2000): Experiments: Planning, Analysis, and Parameter Design Optimization. New York: John Wiley \& Sons.

[13] Ahmadi, M., Vahabzadeh, F., Bonakdarpour, B., Mofarrah, E., Mehranian, M. (2005). Application of the central composite design and response surface methodology to the advanced treatment of olive oil processing wastewater using Fenton's peroxidation. Journal of Hazardous Materials 123 (1-3), 187-195. 
[14] Barwick, V.J., Ellison, S.L.R., Lucking, Ch.L., Burn, M.J. (2001). Experimental studies of uncertainties associated with chromatographic techniques. Journal of Chromatography A 918, 267-276.

[15] Park, S.H., Kim, H.J., Cho, J.I. (2008). Optimal Central Composite Designs for Fitting Second Order Response Surface linear Regression Models. $<$ www.springerlink.com/index/vx742p4850n7h274.pdf $>$

[16] Rigas, F., Panteleos, P., Laoudis, C. (2000). Central Composite Design in a Refinery's Wastewater Treatment by Air Flotation. Global Nest: the Int. J. 2 (3), 245-253.

[17] Varesio, E., Gauvrit, J.Y., Longeray, R., Lantéri, P., Veuthey, J.L. (1997). Central composite design in the chiral analysis of amphetamines by capillary electrophoresis. Electrophoresis 18 (6), 931-937.

[18] ISO GUM (1995). Guide to the expression of uncertainty in measurement, BIPM, IEC, IFCC, ISO, IUPAC and OIML, ISBN 92-67-10188-9, Second Edition

[19] Palenčár, R (2006). Rozdelenie pravdepodobnosti chýb meradiel. (in Slovak) Metrológia a skúšobníctvo 11 (2), 9-12.

[20] Welch, B.L. (1947): The Generalization of Student's Problem when Several Different Population Variances are Involved. Biometrika 34, 28.

Received September 10, 2012. Accepted January 25, 2013. 